fragments over time, as they recover from the effect of land transformation ${ }^{2}$. According to Chase and colleagues, the degree of decay in diversity and species abundance found between large and small fragments is smaller in the older or 'softly' transformed European landscapes than in the more recently and dramatically transformed North American ones. This indicates that, over time, species moving in from the edges of the human-altered habitats might compensate, at least in part, for the ecological functions carried out by native species in larger habitats, causing small fragments to reach a new - yet different - ecological balance.

Although this work underscores the key role of habitat area in maintaining ecosystem processes, there is little exploration of how these processes are altered by habitat loss. Species from higher trophic levels (the upper levels of the food chain), such as predators, require larger areas to maintain their populations compared with species from lower trophic levels, so the number of individuals supported by smaller habitat fragments might not suffice to maintain populations of top predators or consumers, and hence would produce shorter food chains and alter the ecosystem structure ${ }^{15}$. Differences in extinction rates between trophic levels can cause striking changes in ecosystem functioning at habitat edges ${ }^{16}$, jeopardizing the functioning and ecosystem-service provision as natural habitats diminish in size ${ }^{11}$.

Chase and colleagues' results call for a reconsideration of the debate over whether a single large area devoted to conservation would preserve more species than would several small ones that combine to make up the same total size ${ }^{17}$. Some current evidence suggests that one continuous habitat might host fewer species than do many small patches that total the same area ${ }^{18}$. However, the large ecological changes that these small fragments might undergo could end up resulting in massive reductions in ecosystem function and, ultimately, increased extinction rates of native species over the long term compared with the case for a single, large protected area.

Chase and colleagues' approach is good for providing a general overview of the extent of these effects, but to understand exactly how ecological processes are changing locally, a higher level of detail will be needed. This will require going beyond the studies of trophic chains ${ }^{14,16}$ to assess more-complex food webs ${ }^{15}$, and to gather information on changes in species' functional responses and trait diversity in increasingly smaller habitats. Ultimately, this information will reveal which ecological processes are decaying, and what the consequences of such ecosystem decay are for the maintenance of fully functional biodiversity.
Joaquín Hortal is in the Department of Biogeography and Global Change, Museo Nacional de Ciencias Naturales, Spanish National Research Council, Madrid 28006, Spain. Ana M. C. Santos is in the Department of Ecology, Autonomous University of Madrid, Madrid 28049, Spain, and at the Centro de Investigación en Biodiversidad y Cambio Global, Autonomous University of Madrid. e-mails: jhortal@mncn.csic.es;

ana.margarida.c.santos@gmail.com

1. Chase, J. M., Blowes, S. A., Knight, T. M., Gerstner, K. \& May, F. Nature 584, 238-243 (2020).

2. Newbold, T. et al. Nature 520, 45-50 (2015).

3. World Conservation Monitoring Centre. Global

Biodiversity: Status of the Earth's Living Resources 199 (Chapman \& Hall, 1992).

4. Díaz, S. et al. (eds) The Global Assessment Report on Biodiversity and Ecosystem Services: Summary for Policymakers (IPBES, 2019).

5. Rosenzweig, M. L. Species Diversity in Space and Time
(Cambridge Univ. Press, 1995).

6. Arrhenius, O. J. Ecol. 9, 95-99 (1921)

7. Wright, D. H. Oikos 41, 496-506 (1983).

8. Di Marco, M., Venter, O., Possingham, H. P. \& Watson, J. E. M. Nature Commun. 9, 4621 (2018).

9. Chase, J. M. et al. Front. Biogeogr. 11, e40844 (2019).

10. Simons, N. K. et al. Agric. Ecosyst. Environ. 237, 143-153 (2017).

11. Dobson, A. et al. Ecology 87, 1915-1924 (2006).

12. Fischer, J. \& Lindenmayer, D. B. Global Ecol. Biogeogr. 16, 265-280 (2007).

13. Tilman, D., May, R. M., Lehman, C. L. \& Nowak, M. A Nature 371, 65-66 (1994).

14. Didham, R. K., Lawton, J. H., Hammond, P. M. \& Eggleton, P. Phil. Trans. R. Soc. B 353, 437-451 (1998),

15. Holt, R. D. in The Theory of Island Biogeography Revisited (eds Losos, J. B. \& Ricklefs, R. E.) 143-185 (Princeton Univ. Press, 2010).

16. Harrison, M. L. K. \& Banks-Leite, C. Conserv. Biol. https://doi.org/10.1111/cobi.13438 (2019).

17. Simberloff, D. S. \& Abele, L. G. Science 191, 285-286 (1976).

18. Fahrig, L. Glob. Ecol. Biogeogr. 29, 615-628 (2020).

\title{
Mechanobiology
}

\section{Stretch exercises for stem cells expand the skin}

\author{
Matthias Rübsam \& Carien M. Niessen
}

Stretching the skin of mice reveals that mechanical strain

is communicated by a subpopulation of stem cells that proliferate and promote mechanical resistance, and so generate extra skin. See p.268

The cells of our bodies are exposed to a range of mechanical forces - including compression, shear and stretching - that they must resist to maintain tissue integrity and function. For example, skin responds to stretching forces by expanding. Physicians have exploited this particular response for more than 60 years ${ }^{1}$, implanting stretching devices in the skin to cause tissue expansion for plastic surgery or to repair birth defects ${ }^{2}$. But exactly how mechanical strain creates extra tissue in a living organism has not been known. On page 268 , Aragona et al. ${ }^{3}$ now provide compelling insights (at the molecular, single-cell and cell-population level) into how stem cells in the skin of mice sense and communicate stretch to make new tissue.

The surface of the skin - a multi-layered tissue called the epidermis - protects organisms against dehydration and environmental stresses, including mechanical challenges. To ensure lifelong protection, the epidermis is constantly renewed through the generation of new stem cells in its basal layer. This renewal is balanced with differentiation and the movement of stem cells to generate the upper, barrier-forming layers of the epidermis. Ultimately, the barrier-forming cells are shed from the surface, to be replaced by new cells.

Aragona et al. set out to examine how the epidermis responds to strain. The group positioned a device used in human surgeries - a self-inflating gel - under the skin of mice. They then examined indicators of force perception, including changes in cell shape, the structure of a mechanosensitive protein called $\alpha$-catenin, and a network of keratin proteins that provides cells with mechanical resilience. This analysis revealed that epidermal stem cells do indeed sense and respond to strain. The authors observed a temporary increase in stem-cell division, followed by thickening of the epidermis. Thus, increased stem-cell renewal fuels stem-cell differentiation. The two effects combine to maintain a functional barrier at the same time as extra skin is generated.

The researchers next genetically engineered cells in the basal epidermal layer such that the stem cells and their descendants were fluorescently marked. Tracking of these cell lineages over time confirmed that stretching tips the renewal-differentiation balance in favour of making more stem cells. This explains why the epidermis expands in response to stretching. 
Aragona et al. demonstrated that force changes stem cells at the molecular level in several ways. First, stretching increased the expression of genes involved in cell-cell adhesion, which have been shown to communicate force in vitro ${ }^{4}$. Second, expression of components of the actomyosin cytoskeletona network of protein filaments that generates contractile forces in cells ${ }^{5}$ - was increased. Third, stretching promoted signalling through the EGF-Map kinase-ERK pathway (a cascade of proteins that promotes growth). The researchers also assessed changes in chromatin, the DNA-protein complex that parcels up the genome in cells; such changes can lead to altered gene expression. This analysis revealed that stretch induced the expression of a network of regulatory genes that links stem-cell proliferation to skin maintenance.

The authors then examined how strain alters gene-expression profiles of single epidermal stem cells, by sequencing the cells' RNA. This revealed that only a subpopulation of stem cells undergoes the molecular changes associated with a stretched state. Why might this be? Perhaps those that take on the stretched state experience greater force. Alternatively, maybe stem cells exist in varying biochemical states, and thus are more or less sensitive to force. Or local differences in stem-cell shape and mechanics could determine how each cell responds to stretch. Answering this question will require measurements of cellular forces and stiffness in vivo, which is still a major challenge. In addition, it remains unclear whether the stretched stem cells alone are driven to proliferate - or whether these cells then induce expansion of surrounding stem cells.

Aragona et al. next genetically engineered mice to lack Diaph3 and $M y h 9$, genes involved in regulation of the actomyosin cytoskeleton. Without these genes, stem-cell responses to stretch were absent, leading to a barrier defect in the animals. The group observed similar effects in animals engineered to lack the genes encoding YAP and TAZ, and/or in which MAL was inhibited - these three transcription factors normally move to the nucleus to regulate gene expression in response to mechanical signals ${ }^{6}$. Next, the authors examined YAP, TAZ and MAL in mice lacking Diaph3 and Myh9. The transcription factors did not move to the nucleus in response to stretch in these animals. Thus, in normal skin, stretch reorganizes the actomyosin cytoskeleton to promote entry of YAP, TAZ and MAL into the nucleus. These proteins then coordinate transcriptional programs that promote skin growth and barrier formation (Fig. 1).

Finally, the researchers inhibited MAL or the EGF-pathway component ERK in their animals. Inhibition of either protein blocked stem-cell proliferation, but only MAL inhibition led to loss of the 'stretched' molecular state in a

\section{a Normal}

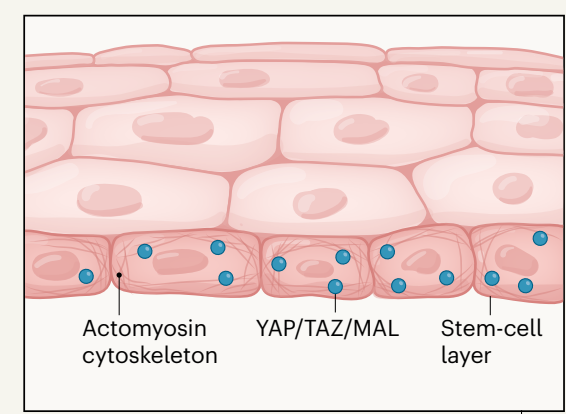

(1) b Stretched
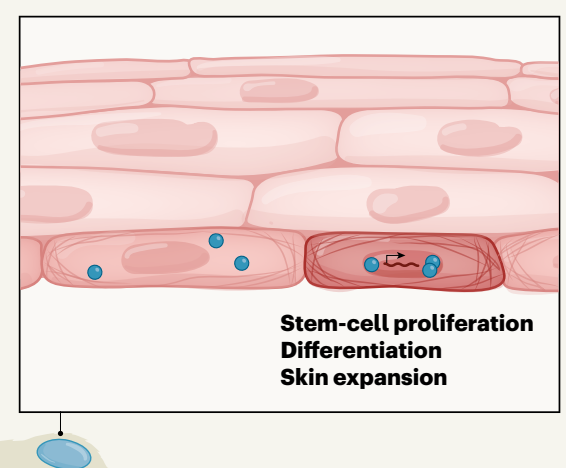

$\mathrm{Ge}$

Figure 1 | How skin stem cells respond to stretching. a, The surface of skin is a multi-layered tissue called the epidermis, which has stem cells in its basal layer. Like all cells, the stem cells have a contractile network of protein filaments called the actomyosin cytoskeleton, and express the transcription factors YAP, TAZ and MAL. b, Aragona et al. ${ }^{3}$ placed an expanding gel under the skin of mice. They report that, in a subset of epidermal stem cells, the actomyosin cytoskeleton is reorganized. This somehow triggers movement of YAP, TAZ and MAL to the nucleus. The proteins induce gene-expression changes that promote an increase in both stem-cell proliferation and differentiation into cells that move into the upper layers of the epidermis. This dual response leads to expansion of skin tissue without compromising the barrier function of the epidermis.

subset of stem cells. Thus, MAL regulates variable cell response to strain, whereas both ERK and MAL are necessary to promote the self-renewal of stem cells. Whether ERK is downstream of YAP, TAZ and MAL, or directly activated by the cytoskeleton ${ }^{7}$, and whether EGF-ERK signalling promotes adaptation to strain in the upper epidermal layers to maintain barrier function during skin expansion ${ }^{8}$, remain open questions.

Overall, Aragona and colleagues' data support a model in which stretch is initially sensed by a subset of stem cells. These cells, through cytoskeletal reorganization and changes in gene expression, coordinate stem-cell renewal and differentiation with adaptation to the mechanical force being experienced. This response guarantees that the skin can maintain its protective function while expanding.

The research opens several avenues for future research. First, what is the contribution of other compartments of the skin (such as the upper, barrier-forming epidermal layers, or the thick dermal layer that underlies the epidermis) in sensing and communicating stretch? The authors' analysis of mice lacking YAP or MAL suggests that stretch also induces a cytoskeletal response in differentiated cells of the upper epidermal layers. Stem-cell differentiation and upward movement can trigger renewal of neighbouring stem cells ${ }^{9}$, thus begging the question of whether the cells immediately above the basal layer are also required for stem-cell responses to strain.

Second, in vitro experiments have demonstrated ${ }^{10}$ that chromatin regulation in the nucleus is key to maintaining stem-cell identity and genome integrity under mechanical stress. Aragona and co-workers' skin-expander model will now allow us to explore these mechanisms invivo.

Third, current models of stem-cell renewal postulate that a single stem cell is equally capable of undergoing renewal or differentiation. However, Aragona and colleagues' lineage-tracing experiments revealed that the number of cells derived from one stem cell (called basal-cell clones) tended to be even. This bias towards clones that have even numbers of cells became much more pronounced on stretching. How the stretched state promotes even-numbered clones is unclear.

The authors propose that this bias can be explained by a model in which stem cells exist in two-progenitor units, in which one stem cell is committed to renewal and the other to differentiation. Communication within and between units would balance the loss of cells through differentiation with renewal. The group performed a mathematical comparison, which indicated that the even-numbered-cell bias and clone dynamics they observed were more consistent with a two-progenitor than with a single-progenitor model. However, the jury on this is still out, because a recent study has provided fresh evidence for the one-progenitor mode ${ }^{11}$.

The current work provides a major step forward in our understanding of how force is interpreted at the single-cell level in living 
organisms. Furthermore, it should encourage others to explore the use of mechanical signals to generate extra skin - not only for reconstructive surgery, but also for diseases associated with impaired regeneration.

Matthias Rübsam and Carien M. Niessen are in the Cologne Excellence Cluster on Stress Responses in Aging-associated Diseases (CECAD), University of Cologne, 50931 Cologne, Germany. M.R. is also at the Max Planck Institute for Biology of Aging, Cologne. C.M.N. is also in the Department Cell Biology of the Skin and the Center for Molecular Medicine Cologne, University of Cologne. e-mails: ruebsam@age.mpg.de;

carien.niessen@uni-koeln.de

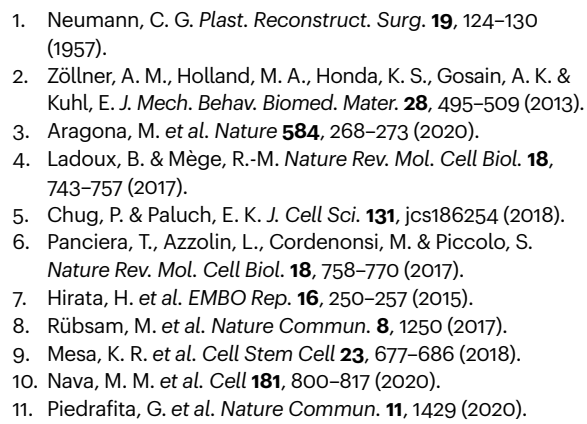

- Piedrafita, G. et al Nature Commun 11 1429 (2020).

This article was published online on 29 July 2020

\section{Biogeochemistry}

\section{Carbon loss from tropical soils increases on warming}

\section{Eric A. Davidson}

Plots of tropical forest soils were warmed by $4{ }^{\circ} \mathrm{C}$ for two years to observe the effects on soil carbon emissions. The increase in efflux of carbon dioxide was larger than expected - a result with worrying implications for climate change. See p.234

The carbon stored in soil could have a big impact on climate change. The global flux of carbon in and out of soils is six to ten times greater than the emissions of carbon dioxide produced as a result of human activities ${ }^{1,2}$. The inputs of carbon to soils from plant detritus (dead wood, leaves and roots) roughly balance the losses to the atmosphere produced by the respiration of soil microorganisms that feed on that material. However, just a $1 \%$ imbalance of global soil-carbon effluxes over influxes would equal about $10 \%$ of global anthropogenic carbon emissions. Carbon in tropical soils was thought to be less vulnerable to loss under climate change than is soil carbon at higher latitudes, but experimental evidence for this was lacking. On page 234, Nottingham et al. ${ }^{3}$ report that tropical-forest soils might be more vulnerable to warming than was thought.

Various soil management practices (such as changes of land use and of tillage methods) can influence the amount of soil carbon present ${ }^{4}$, but climate affects the respiration rate of the microbes that feed on soil carbon, and hence the $\mathrm{CO}_{2}$ efflux from soil ${ }^{5}$. If the net efflux of carbon from soils to the atmosphere increases in a warming world, a positive feedback will accelerate the warming.

Soils are amazingly diverse and differ in several respects that affect microbial respiration $^{6}$. These include: the amount of minerals to which soil carbon can bind and thereby be protected from decomposition by microbial enzymes; water content, which affects the diffusion of carbon to microbial enzymes; the amount, timing and quality of the plant detritus going into the soil; and genetic variation in soil microbial communities. The intrinsic temperature sensitivity of microbial respiration reactions indicated by theory and demonstrated in the laboratory (where other factors are not limiting) therefore often varies from the apparent temperature sensitivity measured in real-world settings ${ }^{5,6}$. Several in situ soil-warming experiments have yielded insights into the effects of temperature on $\mathrm{CO}_{2}$ efflux from soil in temperate and boreal regions ${ }^{6,7}$, but such research is logistically more challenging to implement in tropical forests.

Nottingham and co-workers now present results of a soil-warming experiment (Fig. 1). The authors placed warming rods around the perimeter of undisturbed soil plots in a tropical forest on Barro Colorado Island, Panama, and increased the temperature of the whole soil profile (to a depth of 1.2 metres) by $4{ }^{\circ} \mathrm{C}$ for two years. They measured $\mathrm{CO}_{2}$ efflux using chambers periodically placed over the soil, and observed an unexpectedly large increase (55\%) in soil $\mathrm{CO}_{2}$ emissions. By excluding roots from the soil under some of the chambers, the authors determined that most of the increased $\mathrm{CO}_{2}$ efflux was due to a greater-than-expected increase in the respiration of soil microbes.

The dependence of reaction rates on temperature, including the rates of enzymatic reactions, is described by the Arrhenius equation ${ }^{5}$. According to this equation, the fractional increase in reaction rate is less for a temperature increase of one degree Celsius at higher temperatures than at lower temperatures. This suggests that the response of soil microbial respiration to temperature changes

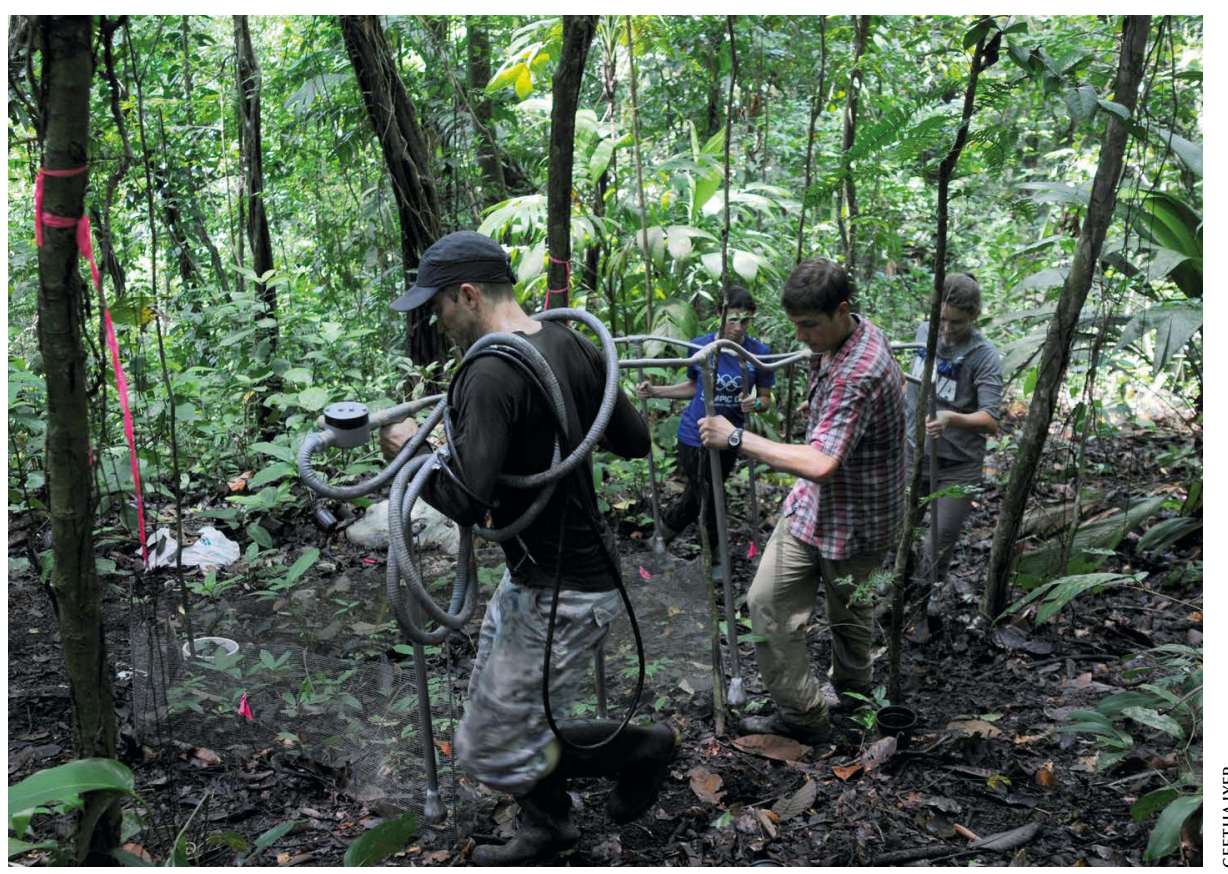

Figure 1 | Preparing to use a heating device in an experiment. Nottingham et al. ${ }^{3}$ buried heating equipment in plots in a topical forest in Panama and monitored the affect of warming on carbon dioxide release from the soil. 